\title{
Molecular Differences Between Squamous Cell Carcinoma and Adenocarcinoma Cervical Cancer Subtypes: Potential Prognostic Biomarkers
}

\author{
Alma Campos-Parra \\ INCan: Instituto Nacional de Cancerologia \\ Milagros Perez-Quintanilla \\ INCan: Instituto Nacional de Cancerologia \\ Antonio Daniel Martínez-Gutierrez \\ INCan: Instituto Nacional de Cancerologia \\ Delia Pérez-Montiel \\ INCan: Instituto Nacional de Cancerologia \\ Oliver Millan-Catalan \\ INCan: Instituto Nacional de Cancerologia \\ Carlos Perez-Plasencia ( $\nabla$ carlos.pplas@gmail.com ) \\ Instituto Nacional de Cancerologia https://orcid.org/0000-0002-8593-8211 \\ David Cantú de León \\ INCan: Instituto Nacional de Cancerologia
}

Research article

Keywords: Cervical Cancer, adenocarcinoma, squamous cell carcinoma, molecular biomarkers, transcript profiling

Posted Date: July 28th, 2021

DOI: https://doi.org/10.21203/rs.3.rs-753668/v1

License: (c) (1) This work is licensed under a Creative Commons Attribution 4.0 International License. Read Full License 


\section{Abstract \\ Purpose}

Cervical cancer (CC) remains a health problem. Persistent infection by high-risk papillomavirus (HR-HPV) is the main cause of this disease. The most frequently diagnosed histological types of $\mathrm{CC}$ are squamous cell carcinoma (SCC) and adenocarcinoma (ADC). Clinically, the prognosis of both types is controversial. Our aim was to search for a molecular profile that distinguishes each histological subtype and predicts the prognosis of one or both subtypes would be of great benefit to these patients.

\section{Methods}

The transcriptome of CC patients from The Cancer Genome Atlas (TCGA) was analyzed using the DESeq2 package to obtain the differentially expressed genes between $A D C$ and SCC. The differentially expressed genes obtained from the TCGA database were validated with an online, publicly available transcriptome dataset (GSE56303) containing data for a Mexican-Mestizo independent cohort. The global biological pathways involving differentially expressed genes between SSC and ADC were obtained by performing an analysis using the Webgestalt platform. In addition, associations of the differentially expressed genes with Overall Survival (OS) were assessed.

\section{Results}

The molecular profile of 70 altered transcripts between ADC and SCC patients from the TCGA cohort was determined. These transcripts were also deregulated in the Mexico-Mestizo cohort with the same Log2FoldChange values. The molecular pathways involved were IL-17, JAK/STAT and Ras signaling. Higher GABRB2 and TSPAN8 expression and lower TMEM40 expression were associated with better OS in the Mexican-Mestizo cohort.

\section{Conclusion}

We were able to detect molecular differences between the ADC and SCC subtypes of CC; however, further studies are required to define the appropriate prognostic biomarker for each histological type.

\section{Introduction}

Despite early screening programs, cervical cancer (CC) is an unresolved health issue. Although its incidence has decreased by approximately $40 \%$, it is currently the second leading cause of cancer-related death in women, accounting for an estimated 270,000 deaths worldwide (1). Unfortunately, in developing countries, most tumors are diagnosed at advanced clinical stages (75-90\%), at which point the tumors 
are often unresponsive to standard treatment; thus, many CC patients die due to disease recurrence or progression [1], [2].

The most frequent histological types of CC are squamous cell carcinoma (SCC), which accounts for approximately $75-90 \%$ of CCs, followed by adenocarcinoma (ADC), which accounts for approximately $10-25 \%$ of all CC cases [3]. It is well described that persistent infection with high-risk papillomavirus (HRHPV) is the causal factor in the development of CC [4]. Strikingly, several reports have described that HPV16 is more frequent in SCCs and HPV18 in ADCs [5], [6], [7]. These histological types are relevant in terms of patient prognosis; according to the National Comprehensive Cancer Network (NCCN) guidelines, the treatment for both is based on concurrent chemoradiotherapy, and the survival outcomes remain uncertain [8]. For example, some studies reported that ADC has a poorer prognosis than SCC patients [9], [10]. For instance, Jung E. and collaborators, in a cohort of 1,133 CC patients reported that the ADC subtype was a significant independent factor for poor overall survival $(O S)(P=0.0001)$. Likewise, Yamauchi $M$. and collaborators reported in a smaller cohort that $A D C$ had a significantly poorer prognosis in $C C$ patients $(P=0.001)$ [11]. On the other hand, other studies reported that $A D C$ showed recurrence and survival rates equivalent to those of SCC patients [12], [13]. For instance, Winer I. and collaborators reported in a small cohort of patients that the 5-year OS was comparable for ADC and SCC $(98.2 \%$ and $95.2 \%$, respectively, $P=0.369)$ [13]. Additionally, in a larger cohort of patients, Xie X. and collaborators reported that there was no difference in the 5 -year survival rate between SCC and ADC patients (87.3\% vs. 82.4\%; $P>0.05)[14]$.

Considering these contradictory data, it is important to find molecular differences that distinguish each subtype based on the gene expression profile and that can be used as prognostic biomarkers for these patients. Some attempts have been made to find molecular differences between ADC and SCC; for instance, Lin W. et al. identified 1733 genes that distinguish ADC from SCC in the lung, esophagus and cervix; however, they mentioned that it is only a catalog of genes and markers that should be studied further [13]. In another study, using microarrays and RT-qPCR assays, Chao A. et al. identified a group of 4 genes (CEACAM5, TACSTD1, S100P and MSLN) that were overexpressed in ADC vs SCC; in this study, CEACAM and TACSTD1 were prognostic factors, showing that it is conceivable to describe different gene profiles for ADC and SCC [15].

Despite the molecular differences between ADC and SCC mentioned above, the evidence is not conclusive, and this field needs to be explored further. In the present work, our major aim was to identify molecular profiles that distinguish ADC and SCC that may have predictive value. To achieve this, we downloaded transcriptome data corresponding to 309 CC patients from TCGA. We compared ADC vs SCC and identified 1678 differentially expressed genes (DEGs) between the two histological types. These data were validated in a Mexican-Mestizo independent cohort, and the results were consistent with those obtained with TCGA analysis. Moreover, we utilized three different databases to distinguish the possible biological pathways in which these genes are involved, and we noticed that SCC tumors have a higher level of activation of critical cancer pathways, such as IL17, JAK-STAT and Ras, than ADC tumors. Strikingly, of the DEGs, GABRB2 and TSPAN8 were associated with OS in the Mexican-Mestizo cohort. 


\section{Materials And Methods \\ 3.1. Analysis of differential expression}

TCGA data of all CC samples (Stage 1-IV) [16],[17] were downloaded using the Bioconductor package TCGAbiolinks [18]. Differential expression analysis was carried out using the DESeq2 package [19], where an adjusted $\mathrm{P}<0.01$ and a log2FoldChange $>2$ or $<2$ were considered significant. Besides, we analyzed the expression profiles of an independent cohort of 89 Mexico-Mestizo patients diagnosed with LACC, obtained from the publicly available database GEO GSE56303 using the R package GEOquery [2], [20], [21]. This dataset was previously used to obtain an mRNA signature capable of predicting the treatment response in CC patients [2]. The data obtained were normalized using the robust multiarray averaging (RMA) method, and differential expression analysis was carried out using the limma package [22]. An adjusted $\mathrm{P}<0.05$ was considered to indicate a significant difference.

\subsection{CC transcriptome and pathway analyses}

Pathway analysis of the differentially expressed genes of both cohorts (TCGA and Mexican-Mestizo cohort) was assessed using the Webgestalt platform [23], where a $\mathrm{P}<0.05$ was considered to indicate a significant difference.

\subsection{OS analysis}

We obtained the clinical data from the 309 cervical tumors from the TCGA and the 89 patients from the Mexican-Mestizo cohort. For each cohort, patients were divided in two groups: high and low, depending in the median expression of each gene. Next, the OS of each group was evaluated using the Kaplan-Meier method, and the statistical significance of survival differences was determined with the log-rank test. Univariate and multivariate Cox proportional hazards regressions were assessed using the survival package implemented in the $\mathrm{R}$ language. A $\mathrm{P}<0.05$ was considered as significant.

\section{Results}

3.1. Determination of the molecular profile distinguishing $A D C$ and $S C C$ among $C C$ Patients from the TCGA database

Our first aim was to determine molecular differences between SCC and ADC. Thus, we analyzed the transcriptomes of the TCGA CC cohort. This dataset contains information on 260 SCC and 49 ADC tumors from patients of different ethnicities. The clinical characteristics are shown in Table 1. It should be noted that the clinical data of the TCGA cohort only contained the HPV information from 22 patients (7\%) (Supplementary table 1 ), whereas 63 patients (70.78\%) from the Mexican-Mestizo were positive to HPV infection (Supplementary Table 2). 
Table 1

Clinical characteristics of the TCGA cohort

\begin{tabular}{|c|c|c|c|c|}
\hline Characteristics & & Total $(n=309)$ & $\operatorname{SCC}(n=260)$ & $\operatorname{ADC}(n=49)$ \\
\hline \multirow[t]{3}{*}{ Ethnicity } & Hispanic or Latino & $24(7.76 \%)$ & $21(8.07 \%)$ & $3(6.12 \%)$ \\
\hline & Not Hispanic or Latino & $171(55.33 \%)$ & $145(55.76 \%)$ & $26(53.06 \%)$ \\
\hline & Not reported & $114(36.91 \%)$ & $94(36.15 \%)$ & $20(40.81 \%)$ \\
\hline \multicolumn{5}{|l|}{ FIGO Stage } \\
\hline & Stage I & $163(52.75 \%)$ & $129(49.61 \%)$ & $34(69.38 \%)$ \\
\hline & Stage II & $70(22.65 \%)$ & $63(24.3 \%)$ & $7(14.28 \%)$ \\
\hline & Stage III & $46(14.88 \%)$ & $43(16.53 \%)$ & $3(6.12 \%)$ \\
\hline & Stage IV & $21(6.79 \%)$ & $17(6.53 \%)$ & $4(8.16 \%)$ \\
\hline & Not reported & $9(2.91 \%)$ & $8(3.07 \%)$ & $1(2.04 \%)$ \\
\hline
\end{tabular}

The results showed 1678 unique DEGs between SCC and ADC (adj $P<0.05$, log2FoldChange +- 2), where most of the ADC tumors were clustered together (Fig. 1). This finding confirms the existence of molecular differences between the two histological types of cervical tumors. A list of the top DEGs between the two histological types is depicted in Table 2. 
Table 2

Top 15 DEGs in ADC in the TCGA dataset

\begin{tabular}{|llll|}
\hline Over-expressed & \multicolumn{3}{l|}{ Under-expressed } \\
\hline Gene & log2FC & Gene & log2FC \\
\hline REG4 & 8.41 & CRNN & -9.38 \\
\hline TM4SF4 & 8.27 & KRT14 & -8.57 \\
\hline TAAR1 & 7.54 & TMPRSS11B & -8.17 \\
\hline FABP1 & 7.52 & CLEC2A & -8.07 \\
\hline SLC17A4 & 7.42 & SPRR2E & -7.84 \\
\hline ANKRD40CL & 7.32 & TMPRSS11BNL & -7.82 \\
\hline REG1A & 6.77 & LCE3E & -7.78 \\
\hline REG1B & 6.65 & SERPINB12 & -7.78 \\
\hline MGAM2 & 6.63 & KPRP & -7.73 \\
\hline LOC105375166 & 6.57 & LCE3D & -7.66 \\
\hline MUC17 & 6.57 & TMPRSS11A & -7.56 \\
\hline CCL15 & 6.33 & SPRR2G & -7.33 \\
\hline SLC39A5 & 6.26 & KRT6C & -7.31 \\
\hline LOC107105282 & 6.23 & LINC02582 & -7.30 \\
\hline CDH17 & 6.20 & KRT1 & -7.26 \\
\hline Values are expressed in Log2 fold change & \\
\hline
\end{tabular}

\subsection{Validation of the molecular profile obtained from the TCGA database in an independent cohort}

To validate the differential expression profile between SCC and ADC obtained from the TCGA data analysis, we utilized an online, publicly available transcriptome dataset (GSE56303) [2]. This dataset only includes a Mexican-Mestizo population, which comprises 89 CC patients, 81 of whom had SCC and 8 of whom had ADC. The analysis of DEGs between the two histological types revealed a profile of 130 transcripts that separated CC patients into ADC vs SCC subgroups $(P<0.05)$ (Supplementary Table 3 ). Strikingly, the 130 differentially expressed transcripts corresponded to 88 unique genes, of which 52 were overexpressed and 36 were downregulated (Fig. 2). HNF1A-AS1, SPINK1, EPS8L3, PROM1 and TM4SF5 were the most overexpressed genes, whereas CLCA2, CALML3, PKP1, GPR87 and SPRR2A were the most downregulated genes in ADC (Table 3). This finding suggested a histology-driven molecular profile. 
Table 3

Top 15 DEGs in ADC in the Mexican-Mestizo CC dataset

\begin{tabular}{|llll|}
\hline \multicolumn{3}{ll}{ Over-expressed } & \multicolumn{3}{l}{ Under-expressed } \\
\hline Gene & log2FC & Gene & log2FC \\
\hline HNF1A-AS1 & 5.30 & CLCA2 & -4.02 \\
\hline SPINK1 & 4.20 & CALML3 & -3.48 \\
\hline EPS8L3 & 3.97 & PKP1 & -3.47 \\
\hline PROM1 & 3.93 & GPR87 & -3.42 \\
\hline TM4SF5 & 3.86 & TP63 & -3.41 \\
\hline EPS8L3 & 3.86 & SPRR2A & -3.23 \\
\hline MUC13 & 3.83 & SPRR1B & -3.18 \\
\hline USH1C & 3.77 & SERPINB3 & -3.17 \\
\hline CLRN3 & 3.75 & GPR87 & -2.98 \\
\hline HGD & 3.73 & ZNF750 & -2.88 \\
\hline MGAM2 & 3.66 & SERPINB4 & -2.86 \\
\hline HGD & 3.63 & S100A9 & -2.85 \\
\hline TSPAN8 & 3.48 & TP63 & -2.84 \\
\hline ARSL & 3.31 & BTBD11 & -2.75 \\
\hline HNF4A & 3.25 & KRT6C & -2.73 \\
\hline Values are expressed in Log2 fold change \\
\hline
\end{tabular}

Notably, of the 88 unique genes differentially expressed between ADC and SCC patients in the MexicanMestizo cohort, 70 were also differentially expressed in the TCGA cohort and even presented the same log2FoldChange values (Fig. 3) (Supplementary Table 4). These findings show a high concordance of the molecular differences of the two histological types between cohorts.

\subsection{Signaling pathway enrichment analysis in DEGs between ADC and SCC patients}

Once we recognized that there was a differential expression profile between SCC and ADC, we decided to determine which signaling pathways the DEGs were involved in. For this purpose, we performed an analysis using the Webgestalt platform exploiting three different databases, the Kyoto Encyclopedia of Genes and Genomes (KEGG), WikiPathways and Reactome databases, to obtain a global pathway 
assessment of the deregulation of both subtypes. It is important to mention that each database has a different set of manually curated genes; thus, the results could differ between them. In the KEGG database analysis, the pathways with the highest number of deregulated genes were the cytokinecytokine receptor interaction, estrogen signaling and IL-17 signaling pathways. Interestingly, we also found that the JAK-STAT signaling pathway was significantly enriched in this analysis (Fig. 4A). When assessing the WikiPathways database, we found that Ras signaling was highly enriched in DEGs (Fig. 4B). In the Reactome database analysis, we also found deregulation in pathways associated with the immune response, such as cytokine- cytokine receptor interaction, and again, estrogen signaling pathway, the IL-17 and JAK-STAT signaling pathways (Fig. 4C). Interestingly, our results show that SCC tumors have a higher level of activation of critical cancer pathways, such as IL17, JAK-STAT and Ras, than ADC tumors (Fig. 4A, B and C). Overall, we assessed different databases and observed enrichment of multiple signaling pathways that are commonly dysregulated in cancer, suggesting that our results are highly reliable and independent of the database used.

To determine whether the DEGs between SCC and ADC are correlated with OS, we used survival data from the TCGA and Mexican-Mestizo cohorts. The Kaplan-Meier curves showed no significant difference between the survival of SCC vs ADC patients in the TCGA dataset (Supplementary Fig. 1). However, when we used the survival data from the Mexican-Mestizo cohort, the Kaplan-Meier analysis showed that high expression of the DEGs GABRB2 and TSPAN8 and underexpression of TMEM40 were associated with favorable OS (Fig. 5). Moreover, the univariate and multivariate cox-regressions using these genes, HPV infection and tumor histology as covariates showed that only TMEM40 and GABRB2 were able to independently differentiate the OS of the patients, whereas HPV infection nor tumor histology were not significant factors for the OS (Table 4.)

Table 4

Univariate and multivariate cox regressions in the Mexican-Mestizo CC dataset

\begin{tabular}{|c|c|c|c|c|c|}
\hline & \multirow[b]{2}{*}{ Overall survival } & \multicolumn{2}{|c|}{ Univariate analysis } & \multicolumn{2}{|c|}{ Multivariate analysis } \\
\hline & & $\mathrm{HR}(95 \% \mathrm{Cl})$ & $\begin{array}{l}\mathrm{P}- \\
\text { value }\end{array}$ & $\mathrm{HR}(95 \% \mathrm{Cl})$ & $\begin{array}{l}\mathrm{P}- \\
\text { value }\end{array}$ \\
\hline TMEM40 & High vs. low expression & $\begin{array}{l}0.27(0.073- \\
0.97)\end{array}$ & 0.045 & $\begin{array}{l}0.32(0.08- \\
1.22)\end{array}$ & 0.096 \\
\hline GABRB2 & & $3.7(1-13)$ & 0.049 & $\begin{array}{l}2.93(0.78- \\
10.9)\end{array}$ & 0.108 \\
\hline TSPAN8 & & $3.5(0.95-13)$ & 0.059 & & \\
\hline HPV & Negative vs. positive & $1.1(0.31-4.1)$ & 0.86 & $\begin{array}{l}1.05(0.28- \\
3.84)\end{array}$ & 0.937 \\
\hline Hystology & $\begin{array}{l}\text { Adenocarcinoma vs } \\
\text { epidermoid }\end{array}$ & $1.2(0.15-9)$ & 0.88 & & \\
\hline
\end{tabular}




\section{Discussion}

It is well described that persistent infection with HR-HPV is the causal factor in the development of CC [4]. Several reports have described that HPV16 is more frequent in SCCs and HPV18 in ADCs [5], [6], [7]. Hence, it is to be expected that there are molecular and clinical differences in both histologic subtypes. In this regard, several reports have described controversial data about the prognosis of CC patients with $A D C$ and SCC. For instance, some of them have reported that $A D C$ is associated with a poorer prognosis than SCC [9], [10]. However, other studies described that ADC and SCC displayed equivalent survival outcomes [11], [12]. In this respect, researchers have focused on identifying molecular differences unique to one or another histological subtype so that they can be used as prognostic biomarkers for these patients. Nonetheless, this field needs to be investigated further. In this work, through the collection of the transcriptome data of all CC patients from the TCGA, we identified a molecular profile of DEGs between ADC and SCC, which was validated in the Mexican-Mestizo independent cohort. Despite the fact that the TCGA database includes patients from different ethnic groups and all clinical stages, we detected that of the DEGs, 70 overlapped with genes from the Mexican-Mestizo database and even displayed the same log2FoldChange values, showing a high concordance in the molecular differences between the two histological types in the datasets. Likewise, Lin W.E. and collaborators analyzed TCGA data and reported 1733 DEGs between ADC and SCC from different tumors, such as those of the esophagus, lung, and cervix [13]. Chao A. and collaborators, through microarray assays, revealed a profile of DEGs between 9 ADC and 16 SCC samples from CC patients; TSPAN-3, CEACAM5, TACSTD1, MSLN and S100P were of particular interest, as they were overexpressed in ADC [15]. These findings suggested that there are molecular differences between ADC and SCC.

The molecular differences between ADC and SCC also have an impact on signaling pathways that promote disease. In this work, the KEGG, WikiPathways and Reactome databases were employed to elucidate these differences. We found that SCC tumors have a higher level of activation of critical cancer pathways, such as IL-17, JAK/STAT and Ras signaling, compared to ADC tumors. In this respect, it is well known that IL-17 is the central cytokine of the Th17 response, during persistent infection of the with HRHPV, this response triggers chronic inflammation of long duration and production of IL-17 among other pro-inflammatory cytokines, hence creating a favorable environment for tumor development associated with associated with poor CC prognosis. Interestingly, higher IL-17 expression has been observed in patients infected with HPV 16 and 18 in relation to other genotypes of the virus [24], [25], [26].

Additionally, Punt S. and collaborators, reported that the predominant cell type expressing IL-17 in SCC CC is the neutrophilic granulocyte and directly contribute to tumorigenesis [27]. Moreover, it has been shown that the expression of Th17 cells is related with stage, tumor size, lymph node metastases, and vasoinvasion [28]. Although there are no studies that evaluate the correlation between IL-17 and histological subtypes (ADC and SCC), we observed that SCC has a higher level of IL-17 pathway activation. It is recognized that cytokines can regulate the local immune state of the cervix. For instance, modifications in some cytokines, such as IL-6 and IL-2, and even IL-17 produced by persistent HR-HPV infection can induce hyperplasia of the local vascular epithelium and activation of the JAK/STAT pathway, promoting local epithelial cell proliferation and disease progression [29]. Thus, HR-HPV may 
also have an impact on different components of the JAK/STAT pathway and its inhibitors [30]. Strikingly, the JAK/STAT signaling, which is involve in proliferation, invasion, survival, inflammation, and immunity and has been reported to be altered in CC patients [31], was another pathway enriched in our analysis. There is a connection between JAK/STAT pathway and TH17 cells, since JAK/STAT pathway is necessary for the differentiation of Th cells [32]. The aberrant signaling of JAK/STAT pathway has not been associated with any histological subtype. Given that ADC and SCC have frequently HPV 18 and 16, respectively, it is expected that this pathway is altered in both subtypes, nonetheless we were able to distinguish that it is enriched in the SCC subtype, which is an interesting fact that has yet to be validated. However, Wu S. and collaborators, reported that STAT1 expression, a member of this pathway, was positively correlated with HPV 16 viral load [33]. Therefore, if the SCC subtype has a higher HPV16 load, an increase activation of this pathway is expected. Interestingly, JAK/STAT pathway has become an attractive therapeutic target in modern CC treatment [34]. Nevertheless, clinical trials are needed to determine whether targeted therapy can benefit CC patients. Another pathway enriched in our study was Ras signaling. Aberrant activation of this pathway is common in several cancers, including CC, which often results from the presence of mutations and amplifications of KRAS. For instance, Zou Y. and collaborators, reported that the frequency of the KRAS mutation ranged from $8.0-17.5 \%$ in cervical ADC [35], to absent or rare in SCC, which suggested that KRAS mutations are frequent and might be a driving factor for the development of cervical ADC but not SCC [36], [37]. Although, in our study we observed that Ras pathway has a higher level of activation in SCC than ADC, which may be due to mutations or alterations in other elements of the pathway [35]. Moreover, Both HPV E6 and E7 oncoproteins can alter cellular RAS signaling pathways to induce cancer [38]. Thus, our findings indicate that SCC is more aggressive than $A D C$; however, the candidate pathways and genes for distinguishing the subtypes need to be further verified.

A main focus of this work was to identify potential genes associated with the prognosis of ADC or SCC, and we generated Kaplan-Meier survival curves with the Mexican-Mestizo and TCGA clinical data. We noticed that GABRB2 and TSPAN8 overexpression in ADC was associated with favorable OS, while TMEM40 overexpression in ADC was associated with poor OS in the Mexican-Mestizo CC cohort. There is only one report associating the GABRB2 protein with cancer; however, GABRB2 overexpression is correlated with lymph node metastasis in thyroid cancer [39]. TSPAN8 has been studied more than GABRB2 in cancer, although it has an oncogenic role because its expression is correlated with a poor prognosis in breast cancer [40], renal cell carcinoma [41] and pancreatic cancer [42]. However, its role in $\mathrm{CC}$ has not yet been studied. On the other hand, TMEM40 has been poorly studied in cancer, but a study proposed that this protein plays a crucial role in proliferation and apoptosis via the p53 signaling pathway [43] and may be a potential diagnostic biomarker for bladder cancer [44]. The expression levels of GABRB2, TSPAN8 and TMEM40 were not associated with the OS of TCGA CC patients (Supplementary Fig. 1). In this respect, it is well known that the TCGA database contains incomplete or no information regarding the survival of many patients. This often occurs because almost all of the patients are untreated and thus have no response data and short follow-up periods [16]. It is possible that for this reason, we did not find any association between the expression of these genes and OS. Lin W.E. noted 
that the TCGA database is interesting because it includes patients of different ethnicities; however, although TCGA datasets are generally large, they may not be representative of the general population [13]. Despite this, they reported that in TCGA ADC samples, the increased expression of the TPX2, KIF4A, IGF2BP1, and HSPA6 genes was correlated with poor survival; in contrast, the expression of MS4A1 (CD20), SUSD2, and CX3CL1 was related to a favorable prognosis [13]. On the other hand, the study by Chao A. and collaborators reported that positive expression of CEACAM5 and TACSTD1 was associated with poor OS in ADC CC patients, and they suggested both genes as independent prognostic factors [15]. Overall, it is obvious that when searching for a prognostic biomarker for ADC or SCC, there were inconsistencies in the studies performed by us, Lin W. E. [13], and Chao W. [15]. The candidate genes differed in each study. This may be due to the lack or incompleteness of survival data, as previously mentioned, which can consequently cause variations in the results regarding the associations of genes with OS. However, the MS4A1, SUSD2, CX3CL1, CEACAM5, and TACSTD genes, which were associated with OS in the studies by Lin W.E. [13] and Chao W. [15], were also identified as DEGs in our study (Supplementary table 3). Thus, it is reasonable to assume that there is a consistent molecular profile for each histological subtype. However, the correlation of these genes with the survival or prognosis of patients' needs to be studied meticulously.

\section{Conclusions}

These findings show a high concordance of the molecular differences for ADC vs SCC among independent cohorts; regardless of HPV type, moreover, further studies are mandatory to define an appropriate prognostic biomarker for each histological type.

\section{Declarations}

\section{Conflicts of Interest:}

The authors declare no conflict of interest

\section{Funding}

National Cancer Institute of México

\section{Availability of data and material}

All data are publicly available

\section{Code availability}

All codes are available in Bioconductor package TCGAbiolinks and R package.

\section{Authors' contributions}


Conceptualization, P-P. C. and CdL. D.; methodology, C-P. A.D., P-Q. M., M-G. A.D. and O.M-C.; formal analysis, P-M. D and C-M. J.; writing-original draft preparation, C-P A.D. and P-Q. M.; writing, review and editing, C-P. A.D., P-P. C. and CdL. D.; supervision, P-P. C.

\section{Ethics approval}

Not applicable

\section{References}

1. Bray F, Ferlay J, Soerjomataram I, Siegel RL, Torre LA, Jemal A. Global Cancer Statistics 2018: GLOBOCAN Estimates of Incidence and Mortality Worldwide for 36 Cancers in 185 Countries. CA Cancer J Clin. 2018;68:394-424. doi:10.3322/caac.21492.

2. Fernandez-Retana J, Lasa-Gonsebatt F, Lopez-Urrutia E, Coronel-Martínez J, Cantu De Leon, D, Jacobo-Herrera N, Peralta-Zaragoza O, Perez-Montiel D, Reynoso-Noveron N, Vazquez-Romo R, et al. Transcript Profiling Distinguishes Complete Treatment Responders with Locally Advanced Cervical Cancer. Transl Oncol. 2015;8:77-84. doi:10.1016/j.tranon.2015.01.003.

3. Lee KB, Lee JM, Park CY, Lee KB, Cho HY, Ha SY. What Is the Difference between Squamous Cell Carcinoma and Adenocarcinoma of the Cervix? A Matched Case-Control Study. Int J Gynecol Cancer Off J Int Gynecol Cancer Soc. 2006;16:1569-73. doi:10.1111/j.1525-1438.2006.00628.x.

4. Berrington de González A, Sweetland S, Green J. Comparison of Risk Factors for Squamous Cell and Adenocarcinomas of the Cervix: A Meta-Analysis. Br J Cancer. 2004;90:1787-91. doi:10.1038/sj.bjc.6601764.

5. Hamzi A, Raub S, Isa NM, Zailani HA, Omar B, Abdullah MF, Mohd Amin WA, Noor RM, Ayub MC, Abidin Z, Kassim F, et al. Distribution of HPV Genotypes in Cervical Cancer in Multi- Ethnic Malaysia. Asian Pac J Cancer Prev APJCP. 2014;15:651-6. doi:10.7314/apjcp.2014.15.2.651.

6. Tjalma WaA, Trinh XB, Rosenlund M, Makar AP, Kridelka F, Rosillon D, Van Dam PA, Collas De Souza, S, Holl K, Simon P, et al. A Cross-Sectional, Multicentre, Epidemiological Study on Human Papillomavirus (HPV) Type Distribution in Adult Women Diagnosed with Invasive Cervical Cancer in Belgium. Facts Views Vis ObGyn. 2015;7:101-8.

7. Hadzisejdić I, Simat M, Bosak A, Krasević M, Grahovac B. Prevalence of Human Papillomavirus Genotypes in Cervical Cancer and Precursor Lesions. Coll Antropol. 2006;30:879-83.

8. Rose PG, Java JJ, Whitney CW, Stehman FB, Lanciano R, Thomas GM. Locally Advanced Adenocarcinoma and Adenosquamous Carcinomas of the Cervix Compared to Squamous Cell Carcinomas of the Cervix in Gynecologic Oncology Group Trials of Cisplatin-Based Chemoradiation. Gynecol Oncol. 2014;135:208-12. doi:10.1016/j.ygyno.2014.08.018.

9. Jung EJ, Byun JM, Kim YN, Lee KB, Sung MS, Kim KT, Jeong DH. Cervical Adenocarcinoma Has a Poorer Prognosis and a Higher Propensity for Distant Recurrence Than Squamous Cell Carcinoma. 
Int J Gynecol Cancer Off J Int Gynecol Cancer Soc. 2017;27:1228-36.

doi:10.1097/IGC.0000000000001009.

10. Yamauchi M, Fukuda T, Wada T, Kawanishi M, Imai K, Hashiguchi Y, Ichimura T, Yasui T, Sumi T. Comparison of Outcomes between Squamous Cell Carcinoma and Adenocarcinoma in Patients with Surgically Treated Stage I-II Cervical Cancer. Mol Clin Oncol. 2014;2:518-24. doi:10.3892/mco.2014.295.

11. Winer I, Alvarado-Cabrero I, Hassan O, Ahmed QF, Alosh B, Bandyopadhyay S, Thomas S, Albayrak S, Talukdar S, Al-Wahab Z, et al. The Prognostic Significance of Histologic Type in Early Stage Cervical Cancer - A Multi-Institutional Study. Gynecol Oncol. 2015;137:474-8. doi:10.1016/j.ygyno.2015.02.005.

12. Xie X, Song K, Cui B, Jiang J, Yang X, Kong, B. A Comparison of the Prognosis between Adenocarcinoma and Squamous Cell Carcinoma in Stage IB-IIA Cervical Cancer. Int J Clin Oncol. 2018;23:522-31. doi:10.1007/s10147-017-1225-8.

13. PLOS Genetics Staff Correction. Comparative Transcriptomes of Adenocarcinomas and Squamous Cell Carcinomas Reveal Molecular Similarities That Span Classical Anatomic Boundaries. PLoS Genet. 2017;13:e1007056. doi:10.1371/journal.pgen.1007056.

14. Wright AA, Howitt BE, Myers AP, Dahlberg SE, Palescandolo E, Van Hummelen P, MacConaill LE, Shoni M, Wagle N, Jones RT, et al. Oncogenic Mutations in Cervical Cancer: Genomic Differences between Adenocarcinomas and Squamous Cell Carcinomas of the Cervix. Cancer. 2013;119:377683. doi:10.1002/cncr.28288.

15. Chao A, Wang T-H, Lee Y-S, Hsueh S, Chao A-S, Chang T-C, Kung W-H, Huang S-L, Chao F-Y, Wei M-L, et al. Molecular Characterization of Adenocarcinoma and Squamous Carcinoma of the Uterine Cervix Using Microarray Analysis of Gene Expression. Int J Cancer. 2006;119:91-8. doi:10.1002/ijc.21813.

16. Liu J, Lichtenberg T, Hoadley KA, Poisson LM, Lazar AJ, Cherniack AD, Kovatich AJ, Benz CC, Levine DA, Lee AV, et al. An Integrated TCGA Pan-Cancer Clinical Data Resource to Drive High-Quality Survival Outcome Analytics. Cell. 2018;173:400-16.e11. doi:10.1016/j.cell.2018.02.052.

17. Cancer Genome Atlas Research Network; Albert Einstein College of Medicine; Analytical Biological Services; Barretos Cancer Hospital; Baylor College of Medicine; Beckman Research Institute of City of Hope; Buck Institute for Research on Aging; Canada's Michael Smith Genome Sciences Centre; Harvard Medical School; Helen F. Graham Cancer Center \&Research Institute at Christiana Care Health Services; et al. Integrated Genomic and Molecular Characterization of Cervical Cancer. Nature 2017, 543, 378-84, doi:10.1038/nature21386.

18. Colaprico A, Silva TC, Olsen C, Garofano L, Cava C, Garolini D, Sabedot TS, Malta TM, Pagnotta SM, Castiglioni I, et al. TCGAbiolinks: An R/Bioconductor Package for Integrative Analysis of TCGA Data. Nucleic Acids Res. 2016;44:e71. doi:10.1093/nar/gkv1507.

19. Love MI, Huber W, Anders S. Moderated Estimation of Fold Change and Dispersion for RNA-Seq Data with DESeq2. Genome Biol. 2014;15:550. doi:10.1186/s13059-014-0550-8. 
20. Fernandez-Retana J, Zamudio-Meza H, Rodriguez-Morales M, Pedroza-Torres A, Isla-Ortiz D, Herrera L, Jacobo-Herrera N, Peralta-Zaragoza O, López-Camarillo C, Morales-Gonzalez F, et al. Gene Signature Based on Degradome-Related Genes Can Predict Distal Metastasis in Cervical Cancer Patients. Tumour Biol J Int Soc Oncodevelopmental Biol Med. 2017;39:1010428317711895. doi:10.1177/1010428317711895.

21. Davis S, Meltzer PS, GEOquery. A Bridge between the Gene Expression Omnibus (GEO) and BioConductor. Bioinforma Oxf Engl. 2007;23:1846-7. doi:10.1093/bioinformatics/btm254.

22. Ritchie ME, Phipson B, Wu D, Hu Y, Law CW, Shi W, Smyth GK. Limma Powers Differential Expression Analyses for RNA-Sequencing and Microarray Studies. Nucleic Acids Res. 2015;43:e47. doi:10.1093/nar/gkv007.

23. Liao Y, Wang J, Jaehnig EJ, Shi Z, Zhang BWebGestalt. 2019: Gene Set Analysis Toolkit with Revamped Uls and APIs. Nucleic Acids Res. 2019, 47, W199-W205, doi:10.1093/nar/gkz401.

24. Vidal AC, Skaar D, Maguire R, Dodor S, Musselwhite LW, Bartlett JA, Oneko O, Obure J, Mlay P, Murphy SK, et al. IL-10, IL-15, IL-17, and GMCSF Levels in Cervical Cancer Tissue of Tanzanian Women Infected with HPV16/18 vs. Non-HPV16/18 Genotypes. Infect Agent Cancer 2015, 10, 10, doi:10.1186/s13027-015-0005-1.

25. Xue J, Wang Y, Chen C, Zhu X, Zhu H, Hu Y. Effects of Th17 Cells and IL-17 in the Progression of Cervical Carcinogenesis with High-Risk Human Papillomavirus Infection. Cancer Med. 2018;7:297306. doi:10.1002/cam4.1279.

26. Mareti Bonin C, Zatorre Almeida-Lugo L, Rodrigues Dos Santos A, Tezelli Junqueira Padovani C, Silva Pina AF, Teixeira Ferreira AM, Dos Santos Fernandes CE, Possati Resende JC, Bovo AC, Tozetti IA. Interleukin-17 Expression in the Serum and Exfoliated Cervical Cells of Patients Infected with HighRisk Oncogenic Human Papillomavirus. Cytokine. 2019;120:92-8. doi:10.1016/j.cyto.2019.04.008.

27. Punt S, Fleuren GJ, Kritikou E, Lubberts E, Trimbos JB, Jordanova ES, Gorter A. Angels and Demons: Th17 Cells Represent a Beneficial Response, While Neutrophil IL-17 Is Associated with Poor Prognosis in Squamous Cervical Cancer. Oncoimmunology. 2015;4:e984539. doi:10.4161/2162402X.2014.984539.

28. Lin W, Zhang H-L, Niu Z-Y, Wang Z, Kong Y, Yang X-S, Yuan F. The Disease Stage-Associated Imbalance of Th1/Th2 and Th17/Treg in Uterine Cervical Cancer Patients and Their Recovery with the Reduction of Tumor Burden. BMC Womens Health. 2020;20:126. doi:10.1186/s12905-020-009720 .

29. Li B, Zhang L, Zhao J, Tan G, Zhang W, Zhang N, Tian J, Qu P. The Value of Cytokine Levels in Triage and Risk Prediction for Women with Persistent High-Risk Human Papilloma Virus Infection of the Cervix. Infect Agent Cancer. 2019;14:16. doi:10.1186/s13027-019-0231-z.

30. Chen J. Signaling Pathways in HPV-Associated Cancers and Therapeutic Implications. Rev Med Virol. 2015;25(Suppl 1):24-53. doi:10.1002/rmv.1823.

31. Campos-Parra AD, Padua-Bracho A, Pedroza-Torres A, Figueroa-González G, Fernández-Retana J, Millan-Catalan O, Peralta-Zaragoza O, Cantú de León, D, Herrera LA, Pérez-Plasencia C. 
Comprehensive Transcriptome Analysis Identifies Pathways with Therapeutic Potential in Locally Advanced Cervical Cancer. Gynecol Oncol. 2016;143:406-13. doi:10.1016/j.ygyno.2016.08.327.

32. O'Shea JJ, Lahesmaa R, Vahedi G, Laurence A, Kanno Y. Genomic Views of STAT Function in CD 4 + T Helper Cell Differentiation. Nat Rev Immunol. 2011;11:239-50. doi:10.1038/nri2958.

33. Wu S, Wu Y, Lu Y, Yue Y, Cui C, Yu M, Wang S, Liu M, Zhao Y, Sun Z. STAT1 Expression and HPV16 Viral Load Predict Cervical Lesion Progression. Oncol Lett. 2020;20:28. doi:10.3892/ol.2020.11889.

34. Valle-Mendiola A, Gutiérrez-Hoya A, Lagunas-Cruz M del Weiss-Steider C, Soto-Cruz B, I. Pleiotropic Effects of IL-2 on Cancer: Its Role in Cervical Cancer. Mediators Inflamm. 2016, 2016, 2849523, doi:10.1155/2016/2849523.

35. Zou Y, Liu F-Y, Wu J, Wan L, Fang S-F, Zhang Z-Y, Luo Y, Chen M-H, Huang M-Z, He M, et al. Mutational Analysis of the RAS/RAF/MEK/ERK Signaling Pathway in 260 Han Chinese Patients with Cervical Carcinoma. Oncol Lett. 2017;14:2427-31. doi:10.3892/ol.2017.6435.

36. Kang S, Kim H-S, Seo SS, Park S-Y, Sidransky D, Dong SM. Inverse Correlation between RASSF1A Hypermethylation, KRAS and BRAF Mutations in Cervical Adenocarcinoma. Gynecol Oncol. 2007;105:662-6. doi:10.1016/j.ygyno.2007.01.045.

37. Wright AA, Howitt BE, Myers AP, Dahlberg SE, Palescandolo E, Van Hummelen P, MacConaill LE, Shoni M, Wagle N, Jones RT, et al. Oncogenic Mutations in Cervical Cancer: Genomic Differences between Adenocarcinomas and Squamous Cell Carcinomas of the Cervix. Cancer. 2013;119:377683. doi:10.1002/cncr.28288.

38. Chakrabarti O, Veeraraghavalu K, Tergaonkar V, Liu Y, Androphy EJ, Stanley MA, Krishna S. Human Papillomavirus Type 16 E6 Amino Acid 83 Variants Enhance E6-Mediated MAPK Signaling and Differentially Regulate Tumorigenesis by Notch Signaling and Oncogenic Ras. J Virol. 2004;78:5934-45. doi:10.1128/JVI.78.11.5934-5945.2004.

39. Jin Y, Jin W, Zheng Z, Chen E, Wang Q, Wang Y, Wang O, Zhang X. GABRB2 Plays an Important Role in the Lymph Node Metastasis of Papillary Thyroid Cancer. Biochem Biophys Res Commun. 2017;492:323-30. doi:10.1016/j.bbrc.2017.08.114.

40. Zhu R, Gires O, Zhu L, Liu J, Li J, Yang H, Ju G, Huang J, Ge W, Chen Y, et al. TSPAN8 Promotes Cancer Cell Stemness via Activation of Sonic Hedgehog Signaling. Nat Commun. 2019;10:2863. doi:10.1038/s41467-019-10739-3.

41. Tang Y, Xie J, Chen Y, Zhang T, Zhuo H, Chen J, Yang P, Cui Q, Zhao L, Li L. Tspan8 Is Highly Expressed in Clear Cell Renal Cell Carcinoma and Indicates Poor Prognosis. Ann Clin Lab Sci. 2020;50:638-44.

42. Heiler S, Wang Z, Zöller M. Pancreatic Cancer Stem Cell Markers and Exosomes - the Incentive Push. World J Gastroenterol. 2016;22:5971-6007. doi:10.3748/wjg.v22.i26.5971.

43. Zhang Z-F, Zhang H-R, Zhang Q-Y, Lai S-Y, Feng Y-Z, Zhou Y, Zheng S-R, Shi R, Zhou J-Y. High Expression of TMEM40 Is Associated with the Malignant Behavior and Tumorigenesis in Bladder Cancer. J Transl Med 2018, 16, 9, doi:10.1186/s12967-017-1377-3. 
44. Zhang Q-Y, Fu M-T, Zhang Z-F, Feng Y-Z, Wei M, Zhou J-Y, Shi R. Expression of TMEM40 in Bladder Cancer and Its Correlation with Clinicopathological Parameters. Int J Clin Exp Pathol. 2017;10:80507.

\section{Figures}
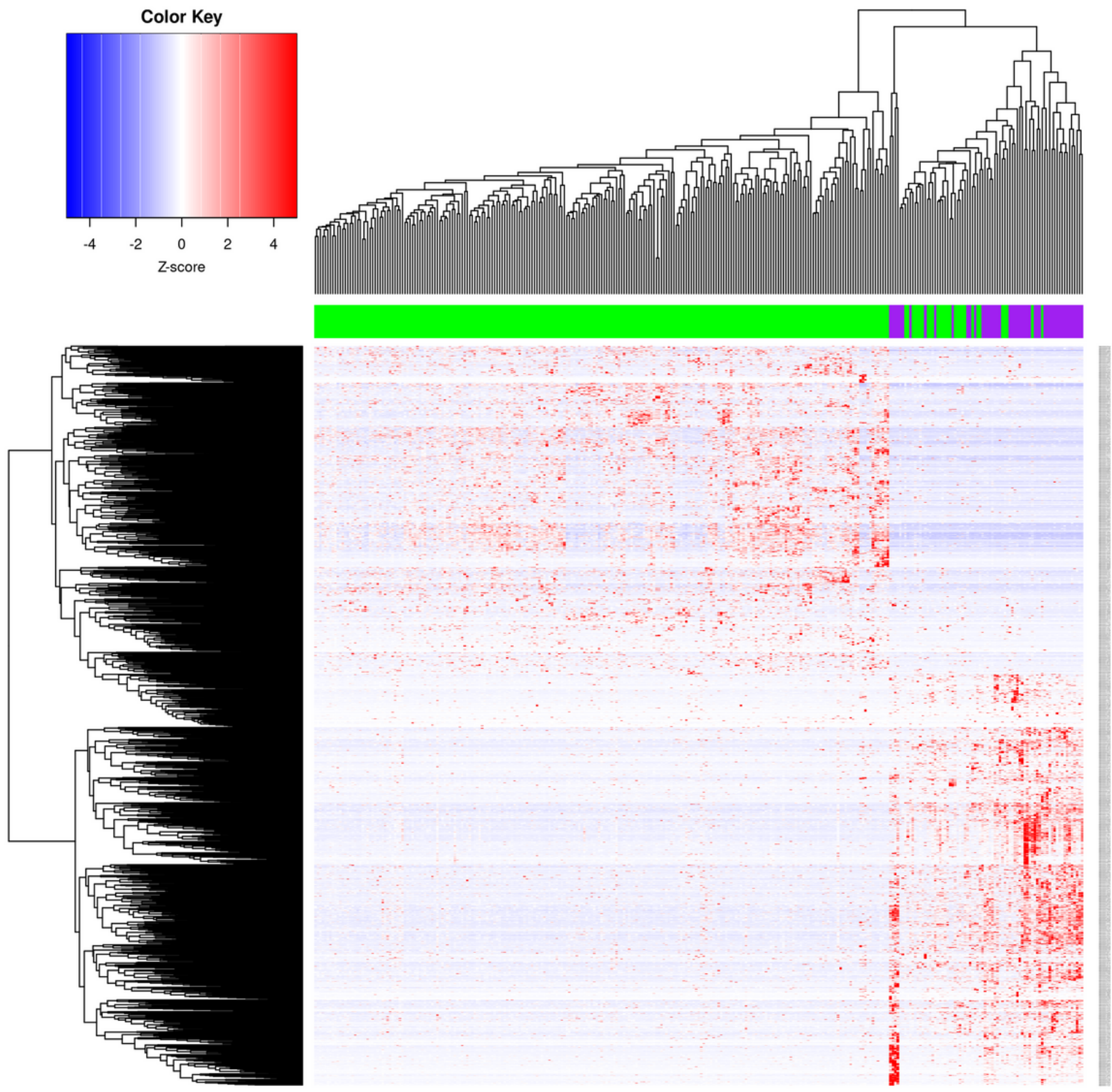

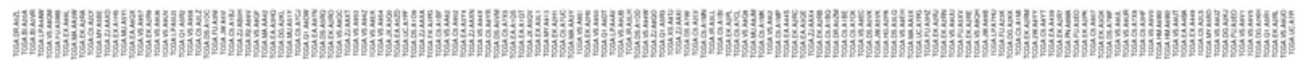

Figure 1 
Molecular profile distinguishing cervical ADC and SCC in patients from the TCGA database. Each column represents a patient with $\mathrm{CC}$, and each row represents the expression of a gene. Gene expression changes are represented in blue (upregulated), red (downregulated) and white (no significant change or the absence of data). There were 1678 unique DEGs between SCC and ADC tumors (adj $P<0.05, \log 2$ fold change +-2), and most of the ADC tumors were clustered together.
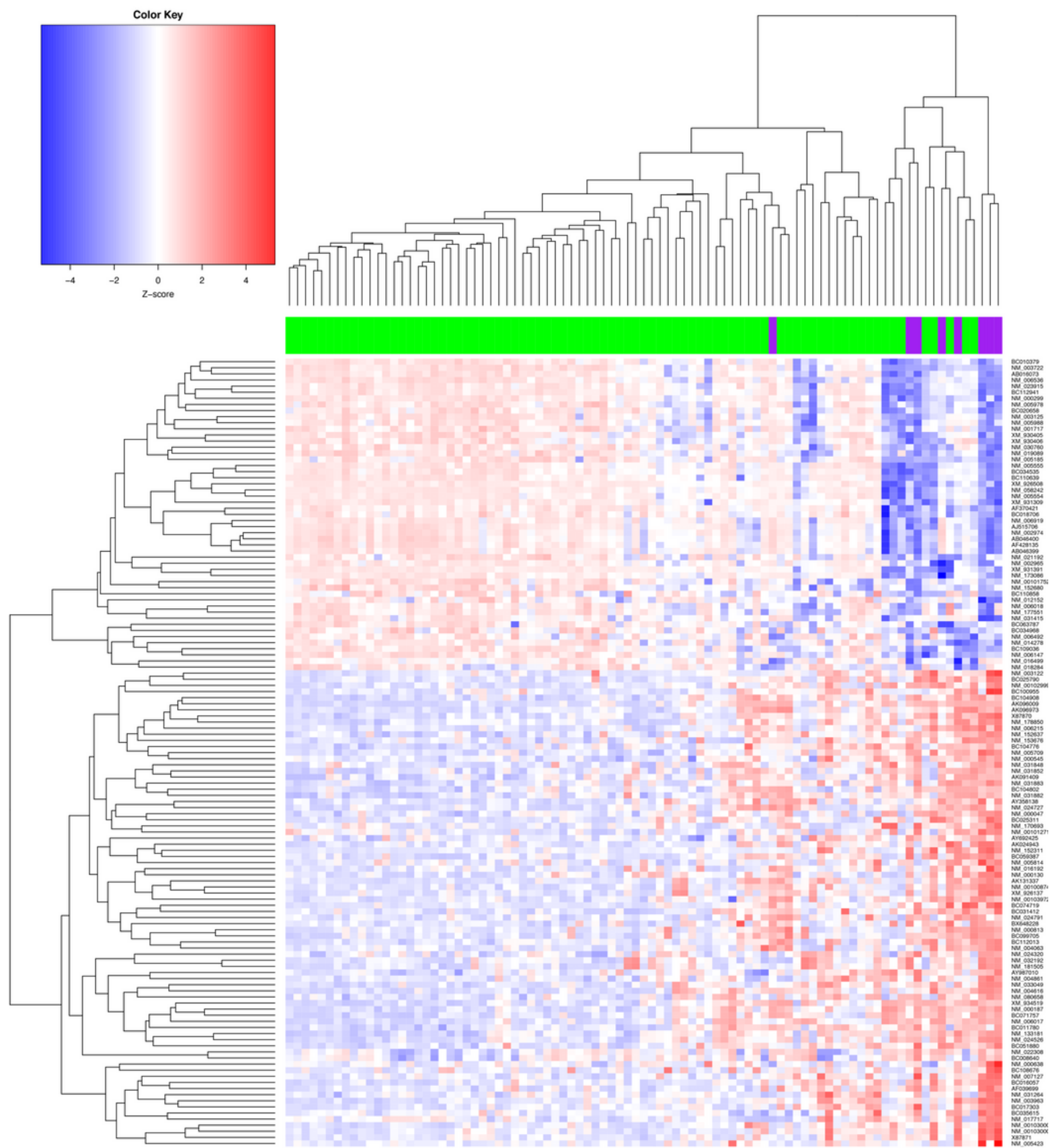

Pmon-mom

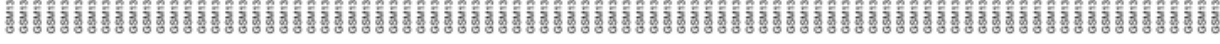

Figure 2 
Molecular profile distinguishing cervical ADC and SCC in patients from the Mexican-Mestizo CC cohort. Each column represents a patient with $\mathrm{CC}$, and each row represents the expression of a gene. Gene expression changes are represented in blue (upregulated), red (downregulated) and white (no significant change or the absence of data). In total, 130 transcripts separated mexican-mestizo CC patients into ADC vs SCC subgroups $(P<0.05)$.

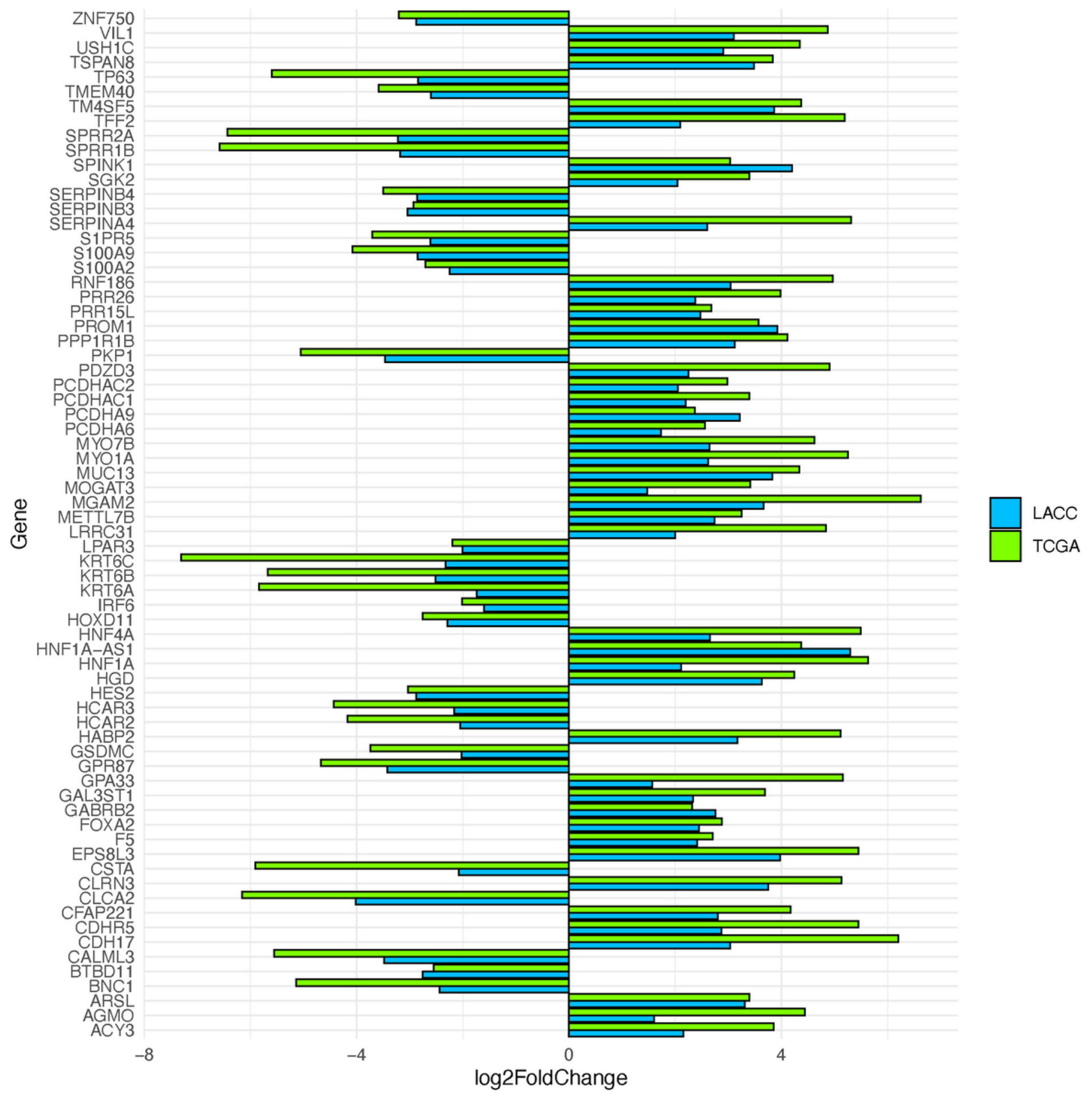

Figure 3 
DEGs in the Mexican-mestizo CC dataset and the TCGA dataset are concordant. Blue represents the 88 unique DEGs in the Mexican-Mestizo CC dataset. Green represents the 70 DEGs from the TCGA dataset.
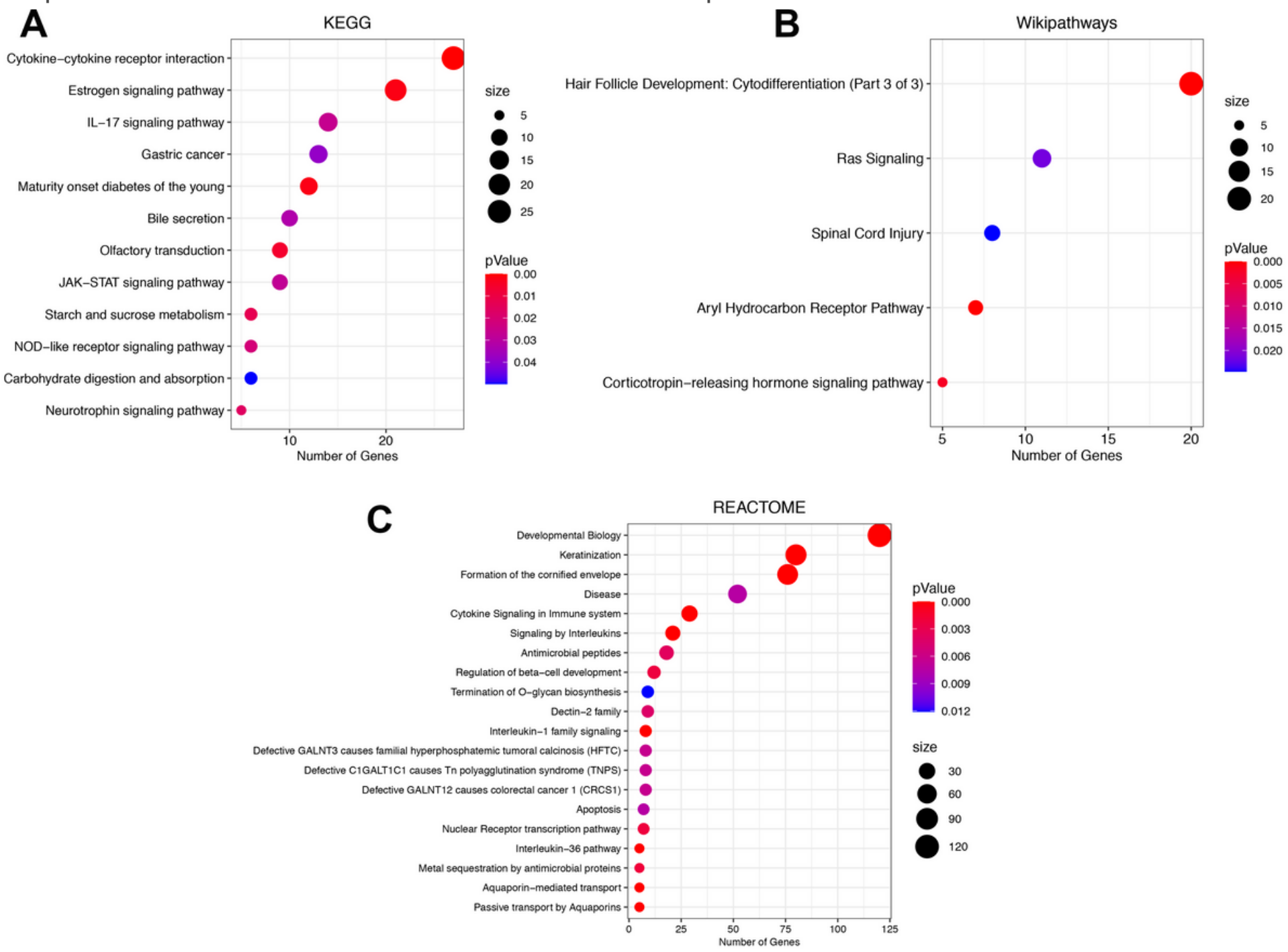

\section{Figure 4}

Signaling pathway enrichment analysis of DEGs between ADC and SCC patients performed with the WebGestalt platform. A) Enriched pathways in the KEGG database analysis. B) Enriched pathways in the WikiPathways database analysis. C) Enriched pathways in the Reactome database analysis. The size of the dots represents the number of DEGs in the pathway, while the dot color represents the significance of the analysis, where red shows a more significantly enriched pathway. 


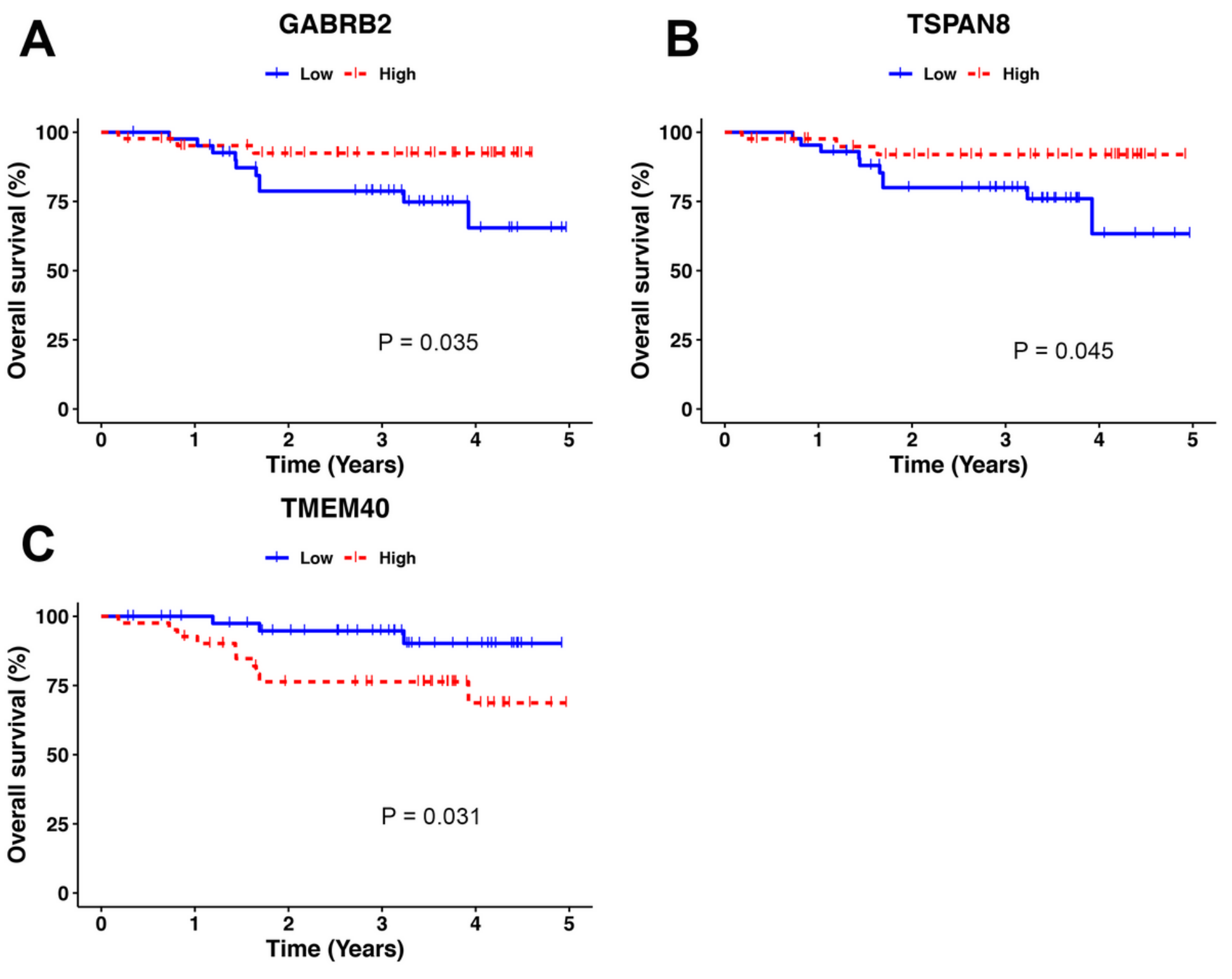

Figure 5

Kaplan-Meier OS analysis of mexican-mestizo patients. A) Differences in the OS of patients with high and low expression of GABRB2. (B) Differences in the OS of patients according to TSPAN8 expression. (C) Differences in the OS of patients according to TMEM40 expression. Blue lines represent patients with low gene expression, and red lines represent patients with high gene expression.

\section{Supplementary Files}

This is a list of supplementary files associated with this preprint. Click to download.

- SupplementaryTable1.xlsx

- SupplementaryTable2.xlsx

- SupplementaryTable3.xlsx 
- SupplementaryTable4.xIsx

- Supplementaryfigure1.tiff 